\title{
Optimal Control Method of Parabolic Partial Differential Equations and Its Application to Heat Transfer Model in Continuous Cast Secondary Cooling Zone
}

\author{
Yuan Wang, Xiaochuan Luo, and Sai Li \\ State Key Laboratory of Synthetical Automation for Process industries, Northeastern University, Shenyang 110004, China \\ Correspondence should be addressed to Xiaochuan Luo; luoxch@mail.neu.edu.cn
}

Received 1 November 2014; Revised 16 December 2014; Accepted 8 January 2015

Academic Editor: Ricardo Weder

Copyright (C) 2015 Yuan Wang et al. This is an open access article distributed under the Creative Commons Attribution License, which permits unrestricted use, distribution, and reproduction in any medium, provided the original work is properly cited.

Our work is devoted to a class of optimal control problems of parabolic partial differential equations. Because of the partial differential equations constraints, it is rather difficult to solve the optimization problem. The gradient of the cost function can be found by the adjoint problem approach. Based on the adjoint problem approach, the gradient of cost function is proved to be Lipschitz continuous. An improved conjugate method is applied to solve this optimization problem and this algorithm is proved to be convergent. This method is applied to set-point values in continuous cast secondary cooling zone. Based on the real data in a plant, the simulation experiments show that the method can ensure the steel billet quality. From these experiment results, it is concluded that the improved conjugate gradient algorithm is convergent and the method is effective in optimal control problem of partial differential equations.

\section{Introduction}

Continuous caster is a machine by which the molten steel is solidified to slabs by spraying on it the cooling water. Because the production situation of secondary cooling zone is very bad, the set point of water volume in secondary cooling plays an important role in the continuous casting [1]. Undercooling of the strand in continuous cast secondary cooling can result in a liquid pool that is too long. However, overcooling can lead to the formation of cracks. The quality of steel billet depends on the behaviour of the surface temperature [2]. Optimal control problem of partial differential equations is encountered [3-5] in many applications ranging from engineering to science. The mathematical model arises in many engineering and physical processes such as heat conduction [2], fluid mechanics [6], and material sciences [7]. Many researchers considered a similar optimal control problem of partial differential equations $[8,9]$ :

$$
\begin{aligned}
\min _{u \in U} J= & \int_{0}^{T}\left[y(0, t)-y_{\text {aim }}(t)\right]^{2} d t \\
& +\alpha \int_{0}^{T}\left[u(t)-u^{*}(t)\right]^{2} d t
\end{aligned}
$$

$$
\begin{array}{ll}
\text { s.t. } & y_{t}-k(x) y_{x x}=0, \quad(x, t) \in(0, l) \times(0, T], \\
& y(x, 0)=\phi(x), \quad x \in(0, l), \\
& k(0) y_{x}(0, t)=u(t), \quad t \in(0, T], \\
& k(l) y_{x}(l, t)=0, \quad t \in(0, T],
\end{array}
$$

where $y$ is temperature (K). $y(0, t)$ is the surface temperature (K). $y_{\text {aim }}$ is the objective temperature. $k(x)$ is a coefficient. $u(t)$ is a controlled variable. $u^{*}(t)$ is a guess for the controlled variable by a priori knowledge. $U=L^{2}(\partial \Omega), \Omega \in(0, l) \times(0, t)$.

$\mathrm{Li}$ [10] applied conjugate gradient algorithm to estimate boundary condition. Lee et al. [11] proposed a repulsive particle swarm optimization algorithm to solve this problem. Farag et al. [12] used a modified partial quadratic interpolation method to solve parabolic optimal control problem. Based on the adjoint problem approach, Kaya and Erdem [13] studied an inverse parabolic problem. Based on the gradient of cost function that is Lipschitz continuous, Hasanov [14] proved existence of a quasisolution of the inverse problem and proposed a monotone iteration scheme. Hasanov [15] 
used the gradient method and proved the convergence of this method. However, the step size in their gradient algorithm is a constant. Step size largely infects the convergent speed of this gradient algorithm. Hasanov and Pektaş [16] considered an inverse source problem. They used a conjugate gradient algorithm to solve this problem, and the good results were obtained.

In this paper, a class of optimal control problems of partial differential equations is abstracted from optimization problem for set-point values in secondary cooling zone process. In our optimal control of partial differential equations problem, the gradient of cost function is proved Lipschitz continuous. We present an improved conjugate gradient algorithm to solve this problem and prove convergence of this algorithm. The simulation experiment shows that this algorithm can effectively solve the optimization problem for set-point values in secondary cooling zone, and the quality of billet is ensured.

\section{Continuous Casting Operation Model of Secondary Cooling Zone}

Continuous casting makes a liquid metal by a special set of cooling devices into a certain section in the shape of a casting process. As shown in Figure 1, $x, y$, and $z$ are the thickness direction of steel billet, the width direction of steel billet, and the length direction of steel billet.

\subsection{Continuous Casting Heat Transfer Model}

2.1.1. Assumptions. Based on the following key assumptions [17], continuous casting heat transfer model can be obtained.

(1) One-dimensional heat transfer is considered, so heat transfer can be ignored in the length and the width direction of billet.

(2) Steel billet at the same cooling zone cools uniformity.

(3) The meniscus surface is assumed to be flat.

(4) In mushy zone, we use the equivalent specific heat instead of specific heat.

(5) The convection heat transfer process in the liquid phase and two-phase zone is equal to conduction heat transfer process.

2.1.2. The Differential Equation. Based on the above assumptions, we can get continuous casting heat transfer model for one-dimensional model.

$$
\frac{\partial y}{\partial t}=k(x) \frac{\partial^{2} y}{\partial x^{2}}
$$

where $y$ is temperature (K). $t$ is times (s). $x$ is slab thickness direction $(\mathrm{m})$ and $k(x)$ is a coefficient depending on physical parameters of the steel, which is calculated by

$$
k(x)=\frac{\lambda}{\rho C_{e}},
$$

where $\rho$ is steel density $\left(\mathrm{kg} / \mathrm{m}^{3}\right), C_{e}$ is specific heat $(\mathrm{J} /(\mathrm{kg} \cdot \mathrm{k}))$, and $\lambda$ is thermal conductivity $(\mathrm{W} / \mathrm{m} \cdot \mathrm{K})$.

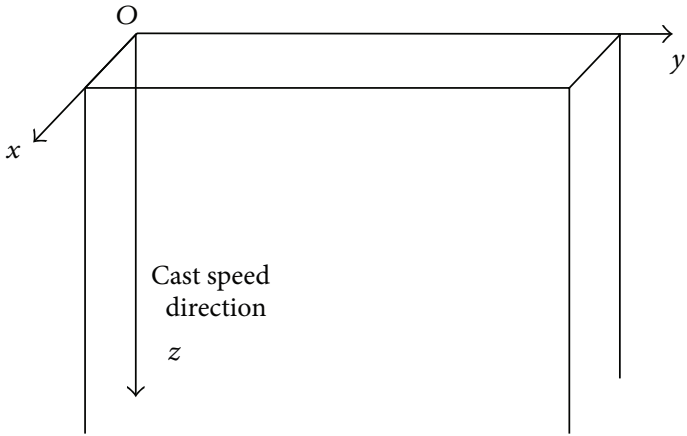

FIGURE 1: Schematic representation of steel slab.

2.1.3. Boundary Conditions. Steel billet goes through the crystallizer, secondary cooling zone, and air cooling zone. The different cooling sections have different cooling characteristics, so the boundary conditions are shown as

$$
k(0) y_{x}(0, t)=u(t),
$$

where $u(t)$ is heat flux in continuous casting process which is calculated by

$$
u(t)=h\left[y(0, t)-T_{w}\right]
$$

where $h$ is the equivalent heat transfer coefficient in secondary cooling zone $\mathrm{W} /\left(\mathrm{m}^{2} \cdot \mathrm{K}\right) . y(0, t)$ is the steel billet surface temperature $(\mathrm{K})$ and $T_{w}$ is cooling water temperature (K):

$$
h=h x\left(\frac{Q_{\text {water }}}{S_{W} S_{L}}\right)^{r w}\left(\frac{Q_{\text {water }}}{S_{W} S_{L}}\right)^{r a}+h r,
$$

where $h x$ and $h r$ are constants. $S_{W}$ is the width of steel billet. $S_{L}$ is the length of steel billet. $Q_{\text {water }}$ is the spray cooling water volume. $Q_{\text {air }}$ is the spray cooling air volume. The boundary condition at $x=l$ can be written as

$$
k(l) y_{x}(l, t)=0 \text {. }
$$

2.2. Optimal Control Model of Continuous Cast Secondary Cooling Zone. Based on continuous casting heat transfer model, we have optimal control model of continuous cast secondary cooling zone:

$$
\begin{array}{ll}
\min _{u \in U} \quad J=\int_{0}^{T}\left[y(0, t)-y_{\mathrm{aim}}(t)\right]^{2} d t \\
\quad+\alpha \int_{0}^{T}\left[u(t)-u^{*}(t)\right]^{2} d t \\
\quad & y_{t}-\frac{\lambda}{\rho C_{e}} y_{x x}=0, \quad(x, t) \in(0, l) \times(0, T], \\
\text { s.t. } & y(x, 0)=y_{0}, \quad x \in(0, l), \\
& k(0) y_{x}(0, t)=h\left[y(0, t)-T_{w}\right], \quad t \in(0, T], \\
& k(l) y_{x}(l, t)=0, \quad t \in(0, T],
\end{array}
$$


where $y$ is steel slab temperature $(\mathrm{K}) . y(0, t)$ is the surface temperature of steel slabs (K). $y_{\text {aim }}$ is the objective temperature of steel slabs $(\mathrm{K})$, which depends on continuous casting technology. $t$ is times (s). $x$ is slab thickness direction (m). $\alpha$ is a positive number. $y_{0}$ is initial casting temperature, which is usually a constant; $U=L^{2}(\partial \Omega), \Omega \in(0, l) \times(0, t)$. Other parameters have already been defined in continuous casting transfer model.

Many researchers $[18,19]$ use $y(0, t)$, the surface temperature of steel billet to evaluate the quality of billet. We hope that the surface temperature $y(0, t)$ is close to the objective temperature $y_{\text {aim }}$ by controlling the boundary condition $u(t)$. In the actual production of continuous casting, the controlled variable cannot be too large, so we also give a penalty for the controlled variable.

According to (3) and (5), $k(x)$ and $u(t)$ in (1) are assigned, so optimal control problem in continuous casting secondary cooling zone transfers to a general optimal control problem in (1). Because of this, we only consider a general optimal control problem in later sections.

\section{Adjoint Problem and Lipschitz Continuity of the Gradient of the Cost Function}

3.1. Adjoint Problem. Based on the first optimization and then discrete method, we can get the adjoint equation firstly and then derive the optimality conditions, using numerical methods to solve this problem. The gradient of the cost function with respect to the control variable can be efficiently calculated by the adjoint problem. Now we give the strong version of the Lagrange function, and then we transfer all constraints into cost function by separate multipliers $p(x, t)$, $p(0, t), p(l, t)$, and $p(x, 0)$ and perform partial integrations with respect to space and time, so the Lagrange function can be obtained:

$$
\begin{aligned}
& L(y, p, x, t) \\
& =\alpha \int_{0}^{T}\left[u(t)-u^{*}(t)\right]^{2} d t \\
& -\int_{0}^{l} \int_{0}^{T} p_{t} y d t d x \\
& -\int_{0}^{T} \int_{0}^{l} k(x) p_{x x}(x, t) y(x, t) d x d t \\
& +\int_{0}^{T}\left[k(l) y_{x}(l, t) p(l, t)\right] d t \\
& +\int_{0}^{T}\left[k(0) y_{x}(0, t) p(0, t)+\left(y(0, t)-y_{\text {aim }}\right)^{2}\right] d t \\
& +\int_{0}^{l} y(x, T) p(x, T) d t \\
& -\int_{0}^{l} \phi(x) p(x, 0) d x \\
& -\int_{0}^{T} u(t) p(0, t) d t .
\end{aligned}
$$

as

The vibration of the function $L(y, p, x, t)$ can be expressed

$$
\begin{aligned}
L & (y+\delta y, p, x, t) \\
= & \alpha \int_{0}^{T}\left[u(t)-u^{*}(t)\right]^{2} d t \\
& +\int_{0}^{l}[y(x, T)+\delta y(x, T)] p(x, T) d t \\
& -\int_{0}^{l} \int_{0}^{T} p_{t}[y(x, t)+\delta y(x, t)] d t d x \\
& -\int_{0}^{T} \int_{0}^{l} k(x) p_{x x}(x, t)[y(x, t)+\delta y(x, t)] d x d t \\
& +\int_{0}^{T} k(0) p_{x}(0, t)[y(0, t)+\delta y(0, t)] d t \\
& +\int_{0}^{T}\left[y(0, t)+\delta y(0, t)-y_{\mathrm{aim}}\right]^{2} d t \\
& +\int_{0}^{T} k(l) p_{x}(l, t)[y(l, t)+\delta y(l, t)] d t \\
& -\int_{0}^{l} \phi(x) p(x, 0) d x \\
& -\int_{0}^{T} u(t) p(0, t) d t .
\end{aligned}
$$

According to (9) and (10), the following equation can be obtained:

$$
\begin{aligned}
\Delta L= & L(y+\delta y, p, x, t)-L(y, p, x, t) \\
= & \int_{0}^{l} p(x, T) \delta y(x, T) d x \\
& -\int_{0}^{l} \int_{0}^{T} p_{t} \delta y(x, t) d t d x \\
& -\int_{0}^{T} \int_{l}^{T} k(x) p_{x x} \delta y(x, t) d x d t \\
& +\int_{0}^{T} k(0) p_{x}(0, t) \delta y(0, t) d t \\
& +\int_{0}^{T}\left[y(0, t)-y_{\text {aim }}(t)\right] \delta y(0, t) d t \\
& +\int_{0}^{T} k(l) p_{x}(l, t) \delta y(l, t) d t .
\end{aligned}
$$

According to (11), the following dual-equation can be obtained:

$$
\begin{gathered}
p_{t}=-k(x) p_{x x}, \quad(x, t) \in(0, l) \times(0, T], \\
p(x, T)=0, \quad x \in(0, l), \\
-k(0) p_{x}(0, t)=\left[y(0, t)-y_{\text {aim }}(t)\right], \quad t \in(0, T], \\
k(l) p_{x}(l, t)=0, \quad t \in(0, T] .
\end{gathered}
$$


Now we obtained adjoint problem in our optimal control problem.

3.2. Lipschitz Continuity of the Gradient of the Cost Function. We can consider the variation of the cost function:

$$
\begin{aligned}
\Delta J(u)= & J(u+\Delta u)-J(u) \\
= & \int_{0}^{T}\left[y(0, t)-y_{\text {aim }}(t)\right] \Delta y(0, t) d t \\
& +\int_{0}^{T}[\Delta y(0, t)]^{2} d t \\
& +\int_{0}^{T}\left[u(t)-u^{*}(t)\right] \Delta u(t) d t+\int_{0}^{T}[\Delta u(t)]^{2} d t .
\end{aligned}
$$

In order to prove that the gradient of the cost function is Lipschitz continuous, we give two other partial differential equations. According to (8), if we let $\Delta y=y(x, t ; u+$ $\Delta u)-y(x, t ; u)$, we obtain the following partial differential equation:

$$
\begin{gathered}
\Delta y_{t}=k(x) \Delta y_{x x}, \quad(x, t) \in(0, l) \times(0, T], \\
\Delta y(x, 0)=0, \quad x \in(0, l), \\
-k(0) \Delta y_{x}(0, t)=\Delta u(t), \quad t \in(0, T], \\
k(l) \Delta y_{x}(l, t)=0, \quad t \in(0, T] .
\end{gathered}
$$

According to (12), the following partial differential equation can be obtained in a similar way:

$$
\begin{gathered}
\Delta p_{t}=-k(x) \Delta p_{x x}, \quad(x, t) \in(0, l) \times(0, T], \\
\Delta p(x, 0)=0, \quad x \in(0, l), \\
-k(0) \Delta p_{x}(0, t)=\Delta y(0, t), \quad t \in(0, T], \\
k(l) \Delta p_{x}(l, t)=0, \quad t \in(0, T] .
\end{gathered}
$$

Lemma 1. For all $\Delta u$, the following identity holds:

$$
\begin{gathered}
\int_{0}^{T}\left[y(0, t ; u)-y_{\text {aim }}(t)\right] \Delta y(0, t) d t \\
=\int_{0}^{T} p(0, t) \Delta u(t) d t .
\end{gathered}
$$

Proof. It is easy to know that the following identity holds:

$$
\begin{aligned}
\int_{0}^{T} & \int_{0}^{l}\left[k(x) p_{x}(x, t) \Delta y(x, t)\right]_{x} d x d t \\
= & \int_{0}^{T} k(l) p_{x}(l, t) \Delta y(l, t) d t \\
& -\int_{0}^{T} k(0) p_{x}(0, t) \Delta y(0, t) d t .
\end{aligned}
$$

Thus boundary conditions of (12) show

$$
\begin{gathered}
\int_{0}^{T}\left[y(0, t ; u)-y_{\text {aim }}(t)\right] \Delta y(0, t) d t \\
=-\int_{0}^{T} k(0) p_{x}(0, t) \Delta y(0, t) d t .
\end{gathered}
$$

The following equations can be obtained:

$$
\begin{aligned}
\int_{0}^{T}[ & \left.y(0, t ; u)-y_{\text {aim }}(t)\right] \Delta y(0, t) d t \\
= & \int_{0}^{T} \int_{0}^{l}\left[k(x) p_{x}(x, t) \Delta y(x, t)\right]_{x} d x d t \\
& -\int_{0}^{T} k(l) p_{x}(l, t) \Delta y(l, t) d t \\
= & \int_{0}^{T} \int_{0}^{l} k(x) p_{x x}(x, t) \Delta y(x, t) d x d t \\
& +\int_{0}^{T} \int_{0}^{l} k(x) p_{x}(x, t) \Delta y_{x}(x, t) d x d t \\
& -\int_{0}^{T} \int_{0}^{l} p(x, t) \Delta y(x, t) d x d t \\
& -\int_{0}^{T} k(0) p(0, t) \Delta y_{x}(0, t) d t \\
= & \int_{0}^{T} p(0, t) \Delta u d t .
\end{aligned}
$$

Lemma 1 can be proved.

Lemma 2. There exists a constant $c>0$, so that the following equation can be obtained:

$$
\int_{0}^{T}|\Delta y(0, t)|^{2} d t \leq c \int_{0}^{T}|\Delta u(t)|^{2} d t .
$$

Proof. If we multiply both sides of (14) by $\Delta y(x, t)$ and integrate on time and space, the following equation can be obtained:

$$
\int_{0}^{T} \int_{0}^{l}\left[\Delta y_{t}-\left(k(x) \Delta y_{x}\right)_{x}\right] \Delta y d x d t=0
$$

It is easy to know that the following identity holds:

$$
\begin{aligned}
\int_{0}^{T} \int_{0}^{l} \Delta y_{t} \Delta y d x d t & =\frac{1}{2} \int_{0}^{T} \int_{0}^{l}\left[(\Delta y)^{2}\right] d x d t \\
& =\frac{1}{2} \int_{0}^{l} \Delta y(x, T)^{2} d x .
\end{aligned}
$$


According to the boundary conditions of (14), the following equation can be derived:

$$
\begin{aligned}
\int_{0}^{l} \int_{0}^{T}\left(k(x) \Delta y_{x}\right)_{x} \Delta y d t d x \\
=\int_{0}^{l} \int_{0}^{T}\left(k(x) \Delta y_{x} \Delta y\right)_{x} d t d x \\
\quad-\int_{0}^{l} \int_{0}^{T} k(x)\left(\Delta y_{x}\right)^{2} d t d x \\
=-\int_{0}^{l} k(0) \Delta y_{x}(0, t) \Delta y(0, t) d t \\
\quad-\int_{0}^{l} \int_{0}^{T} k(x)\left(\Delta y_{x}\right)^{2} d t d x \\
=\int_{0}^{T} \Delta y(0, t) \Delta u(t) d t \\
\quad-\int_{0}^{l} \int_{0}^{T} k(x)\left(\Delta y_{x}\right)^{2} d t d x .
\end{aligned}
$$

Taking (22) and (23) into (21), we have the following equations:

$$
\begin{gathered}
\int_{0}^{T}[\Delta y(x, T)]^{2}+\int_{0}^{l} \int_{0}^{T} k(x)\left(\Delta y_{x}\right)^{2} d t d x \\
=\int_{0}^{T} \Delta y(0, t) \Delta u(t) d t .
\end{gathered}
$$

We use the $\varepsilon$-inequality

$$
\alpha \beta \leq \varepsilon \frac{\alpha^{2}}{2}+\frac{\beta^{2}}{2 \varepsilon}, \quad \forall \alpha, \beta \in R, \forall \varepsilon>0 .
$$

On the right-hand side of (24), then we have

$$
\begin{aligned}
\int_{0}^{T} \Delta u \Delta y(0, t) d t \leq & \frac{1}{2 \varepsilon} \int_{0}^{T}|\Delta u|^{2} d t \\
& +\frac{\varepsilon}{2} \int_{0}^{T}|\Delta y(0, t)|^{2} d t .
\end{aligned}
$$

According to (24) and (26), the following equation can be derived:

$$
\begin{gathered}
\int_{0}^{l} \int_{0}^{T} k(x)\left(\Delta y_{x}\right)^{2} d t d x+\int_{0}^{l}[\Delta y(x, T)]^{2} d t \\
\quad \leq \frac{1}{2 \varepsilon} \int_{0}^{T}|\Delta u|^{2} d t+\frac{\varepsilon}{2} \int_{0}^{T}|\Delta y(0, t)|^{2} d t .
\end{gathered}
$$

From literature [14], the following equation can be obtained:

$$
\begin{aligned}
& 2 l^{2} \int_{0}^{l} \int_{0}^{T}(\Delta y)^{2} d t d x+2 l \int_{0}^{T}[\Delta y(0, t)]^{2} d t \\
& \quad \leq \frac{1}{2 \varepsilon} \int_{0}^{T}(\Delta u)^{2} d t+\frac{\varepsilon}{2} \int_{0}^{T}[\Delta y(0, t)]^{2} d t .
\end{aligned}
$$

So the following equation can be obtained:

$$
\int_{0}^{T}|\Delta y(0, t)|^{2} d t \leq c \int_{0}^{T}|\Delta u(t)|^{2} d t .
$$

We can know that there is a constant $c$ which makes (20) established. Lemma 2 can be proved.

Lemma 3. Consider

$$
\int_{0}^{T}|\Delta y(0, t)|^{2} d t=\int_{0}^{T} \Delta p(0, t) \Delta u(t) d t
$$

Proof. According to (15), the weak solution of this partial differential equation is the following equation:

$$
\begin{gathered}
\int_{0}^{T} \int_{0}^{l}(\Delta p \Delta y)_{t} d x d t-\int_{0}^{T} \int_{0}^{l} \Delta p \Delta y_{t} d x d t \\
+\left.\int_{0}^{T} k(x) \Delta y \Delta p\right|_{x=0} ^{x=l} d t \\
\quad-\int_{0}^{T} \int_{0}^{l} k(x) \Delta p_{x} \Delta y_{x} d x d t=0 .
\end{gathered}
$$

According to the boundary conditions of (14) and (15), the following equation can be obtained:

$$
\begin{aligned}
& \int_{0}^{T} k(l) \Delta y(l, t) \Delta p_{x}(l, x) d t \\
& \quad-\int_{0}^{T} k(0) \Delta y(0, t) \Delta p_{x}(0, x) d t \\
& \quad-\int_{0}^{T} k(l) \Delta y_{x}(l, t) \Delta p(l, x) d t \\
& \quad+\int_{0}^{T} k(0) \Delta y_{x}(0, t) \Delta p(0, x) d t \\
& =\int_{0}^{T}[\Delta y(0, t)]^{2}=\int_{0}^{T} \Delta p(0, t) \Delta u(t) d t .
\end{aligned}
$$

Lemma 3 can be proved.

Theorem 4. If the gradient of cost function is Lipschitz continuous, there is a constant L to make the following equation hold:

$$
\left\|J^{\prime}(u+\Delta u)-J^{\prime}(u)\right\| \leq L\|\Delta u\| .
$$

Proof. Taking into account Lemmas 1, 2, and 3, we can obtain the following equation:

$$
\begin{aligned}
& \left(J^{\prime}(u+\Delta u)-J^{\prime}(u), \Delta u\right) \\
& \quad=\int_{0}^{T} \Delta p(0, t) \Delta u d t \\
& \quad=\int_{0}^{T}[\Delta y(0, t)]^{2} \leq L \int_{0}^{T}[\Delta u(t)]^{2} d t .
\end{aligned}
$$




\section{Conjugate Gradient Algorithm Solves Optimal Control of Parabolic Partial Differential Equations and Its Convergent Analysis}

4.1. Conjugate Gradient Algorithm. After we prove that the gradient of cost function is Lipschitz continuous, we can use the conjugate gradient algorithm to solve this PDE optimization problem. If we choose the reasonable parameters in conjugate gradient algorithm, we can prove the convergence of conjugate gradient algorithm.

As shown in Figure $2, k$ is iteration number. $d_{k}$ is iteration direction. $g_{k}$ is gradient of cost function. $\alpha$ is step size. $u^{k}$ is controlled quantity. $J(u)$ is cost function. $\beta_{k}$ is a parameter in conjugate gradient algorithm, and eps is a small constant.

Because we use FR conjugate method to solve this optimal problem, $d_{k}$ and $\beta_{k}$ can be calculated by the following equations:

$$
\begin{gathered}
d_{k}=-g_{k}+\beta_{k} d_{k-1}, \\
\beta_{k}=\frac{g_{k}^{T} g_{k}}{g_{k-1}^{T} g_{k-1}} .
\end{gathered}
$$

Step size largely infects the convergent speed of conjugate gradient algorithm. If we choose Wolfe line search rules, we have the following formula [20]:

$$
\begin{array}{r}
f\left(x_{k}+\alpha_{k} d_{k}\right) \leq f\left(x_{k}\right)+\sigma_{1} \alpha(k) g_{k}^{T} d_{k}, \\
\left|\nabla f\left(x_{k}+\alpha_{k} d_{k}\right)\right| \geq \sigma_{2}\left|g_{k}^{T} d_{k}\right|, \\
0<\sigma_{1}<\sigma_{2}<\frac{1}{2} .
\end{array}
$$

\subsection{Convergence Analysis of Conjugate Gradient Algorithm}

Theorem 5. If $d_{k}, \beta_{k}$, and $\alpha_{k}$ are calculated by (35) and (36), the following equation holds:

$$
\liminf _{k \rightarrow \infty}\left\|g_{k}\right\|=0
$$

Proof. According to (36), the following equations can be obtained:

$$
\begin{aligned}
-\sum_{i=0}^{k} \sigma_{2}^{i} \leq \frac{g_{k}^{T} d_{k}}{\left\|g_{k}\right\|^{2}} & \leq-2+\sum_{i=0}^{k} \sigma_{2}^{i}, \\
\forall k & =0,1, \ldots, \infty, \\
-\sum_{i=0}^{k} \sigma_{2}^{i}<-\sum_{i=0}^{\infty} \sigma_{2}^{i} & =\frac{1}{1-\sigma_{2}}<2 .
\end{aligned}
$$

According to (38) and (39), the following equation can be obtained:

$$
\left|g_{k}^{T} d_{k-1}\right| \leq-\sigma_{2} g_{k-1}^{T} d_{k-1}<\frac{\sigma_{2}}{1-\sigma_{2}}\left\|g_{k-1}\right\|
$$

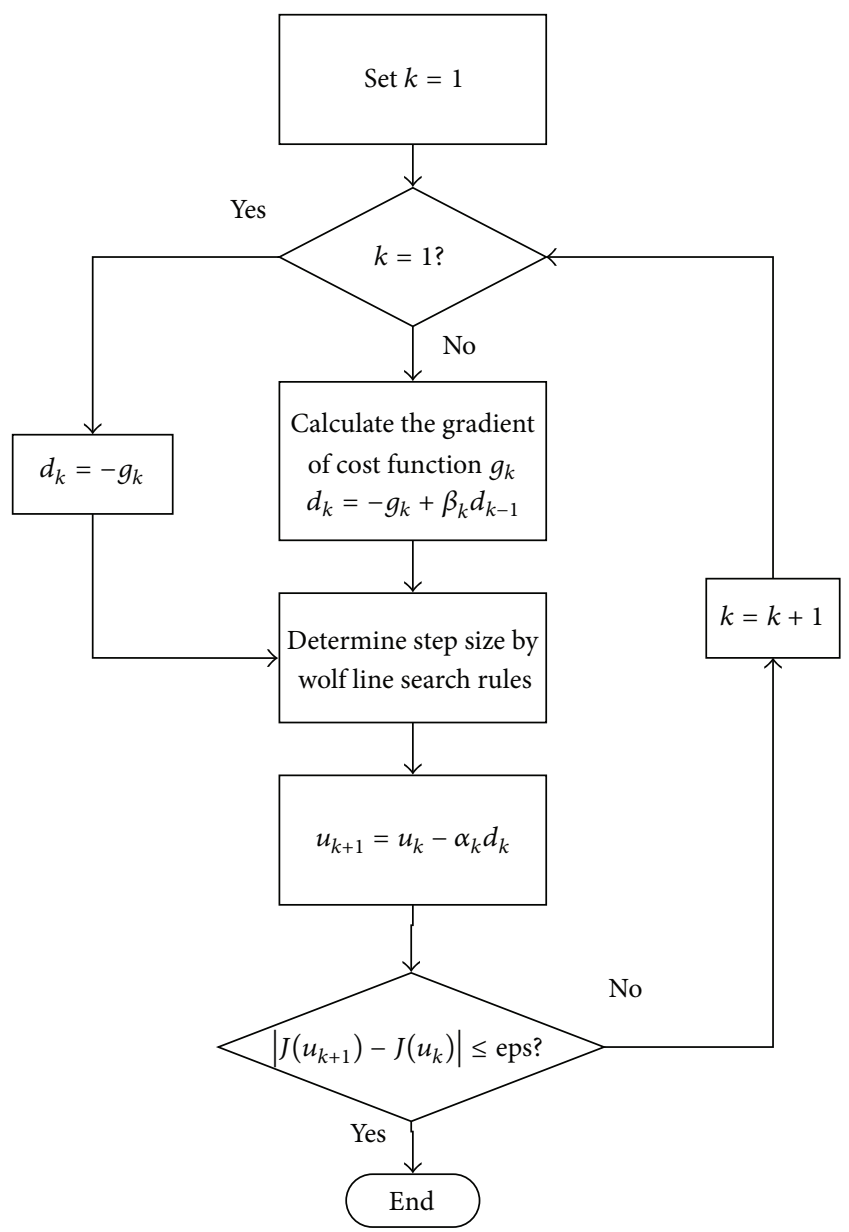

FIGURE 2: Conjugate gradient algorithm process.

According to the recursion formula of $d_{k}$, the following equation can be represented:

$$
\begin{aligned}
\left\|d_{k}\right\|^{2} & =\left\|g_{k}\right\|^{2}-2 \beta_{k-1} g_{k}^{T} d_{k-1}+\beta_{k-1}^{2}\left\|d_{k-1}\right\|^{2} \\
& \leq\left\|g_{k}\right\|^{2}-\frac{2 \sigma_{2}}{1-\sigma_{2}}\left\|g_{k}\right\|^{2}+\beta_{k-1}^{2}\left\|d_{k-1}\right\| \\
& =\frac{1+\sigma_{2}}{1-\sigma_{2}}\left\|g_{k}\right\|^{2}+\beta_{k-1}^{2}\left\|d_{k-1}\right\|^{2} \\
& =\left\|d_{k}\right\|^{2} \leq \frac{1+\sigma_{2}}{1-\sigma_{2}}\left\|g_{k}\right\|^{4} \sum_{i=1}^{k} \frac{1}{\left\|g_{i}\right\|^{2}}+\frac{\left\|g_{k}\right\|^{2}}{\left\|g_{0}\right\|^{2}} .
\end{aligned}
$$

If this algorithm is not convergent, (37) does not hold and there exists a constant $\varepsilon_{0}>0$, so that the following identity holds:

$$
\left\|g_{k}\right\| \geq \varepsilon_{0}, \quad\left\|d_{k}\right\|^{2} \leq \tau_{1}(k+1) .
$$


According to (39), we can obtain the following equations:

$$
\begin{aligned}
& \cos \theta_{k}=\frac{g_{k}^{T} d_{k}}{\left\|g_{k}\right\|\left\|d_{k}\right\|} \\
& \geq\left(2-\sum_{i=0}^{k} \sigma_{2}^{j}\right) \frac{\left\|g_{k}\right\|}{\left\|d_{k}\right\|} \geq \frac{1-2 \sigma_{2}}{1-\sigma_{2}} \frac{\left\|g_{k}\right\|}{\left\|d_{k}\right\|}, \\
& \sum_{k=0}^{\infty} \cos ^{2} \theta_{k} \geq\left(\frac{1-2 \sigma_{2}}{1-\sigma_{2}}\right)^{2} \sum_{k=0}^{\infty} \frac{\left\|g_{k}\right\|^{2}}{\left\|d_{k}\right\|^{2}} \geq \tau_{2} \sum_{k=0}^{\infty} \frac{1}{k+1} .
\end{aligned}
$$

Since $g(x)$ is the gradient of cost function, we have proven that $g(x)$ is Lipschitz continuous. There exists a constant, so that the following inequality holds:

$$
\begin{aligned}
\sigma_{2} g_{k}^{T} d_{k} & \leq g_{k+1}^{T} d_{k}=g_{k}^{T}+\left(g_{k+1}-g_{k}\right)^{T} d_{k} \\
& \leq g_{k}^{T} d_{k}+\alpha_{k} L\left\|d_{k}\right\|^{2} .
\end{aligned}
$$

Then we can obtain the following equations:

$$
\begin{gathered}
\alpha_{k} \geq-\frac{1-\sigma_{2}}{L\left\|d_{k}\right\|^{2}} g_{k}^{T} d_{k}, \\
f_{k+1} \leq f_{k}-\sigma_{1} \frac{1-\sigma_{2}}{L}\left\|g_{k}\right\|^{2} \cos ^{2} \theta_{k} .
\end{gathered}
$$

The sequence $f_{k}$ is monotonic decline and has lower bound, so $\sum_{k=0}^{\infty}\left\|g_{k}\right\|^{2} \cos ^{2} \theta_{k}$ is convergent. Since $\left\|g_{k}\right\| \geq \varepsilon_{0}$, $\sum_{k=0}^{\infty} \cos ^{2} \theta_{k}$ is convergent and we can conclude that $\cos ^{2} \theta_{k}$ is convergent and not convergent at the same time. Thus the assumption $\left\|g_{k}\right\|$ is invalid and we can prove that (37) holds, so this conjugate gradient algorithm is convergent.

4.3. An Optimal Choice of the Stopping in the Conjugate Gradient Algorithm. It is important to understand the behavior of the cost function value depending on the iteration number. For given smooth objective temperature $y_{\text {aim }}$, an optimal control problem is solved by conjugate gradient algorithm:

$$
\begin{gathered}
y_{\text {aim }}=1173.16+100 \sin \left(\frac{\pi}{10} t\right)(\mathrm{K}), \\
k=\frac{7250 \times 540}{50}, \quad \text { eps }=1 \times 10^{-1}, \\
u(x, 0)=1173.16 \mathrm{~K}, \quad L=0.25 \mathrm{~m}, \\
T=20 \mathrm{~s}, \quad u^{*}=0, \quad \alpha=1 \times 10^{-7} .
\end{gathered}
$$

As shown in Figure 3, the behaviour of cost function value as a function of the iteration number consists of three phases: the initial phase of rapid decrease but very short duration, the second phase of slow decrease, and the third phase of almost constant behaviour.

When dealing with optimal control problems, the stopping parameter plays an important role in conjugate gradient algorithm. However, it is difficult to choose a good stopping parameter eps. If stopping parameter eps is too large, the result cannot converge to optimal solution. The iteration number is too large because stopping parameter is too small. As shown in Table 1, the best value of stopping parameter is achieved at eps $=0.1$.

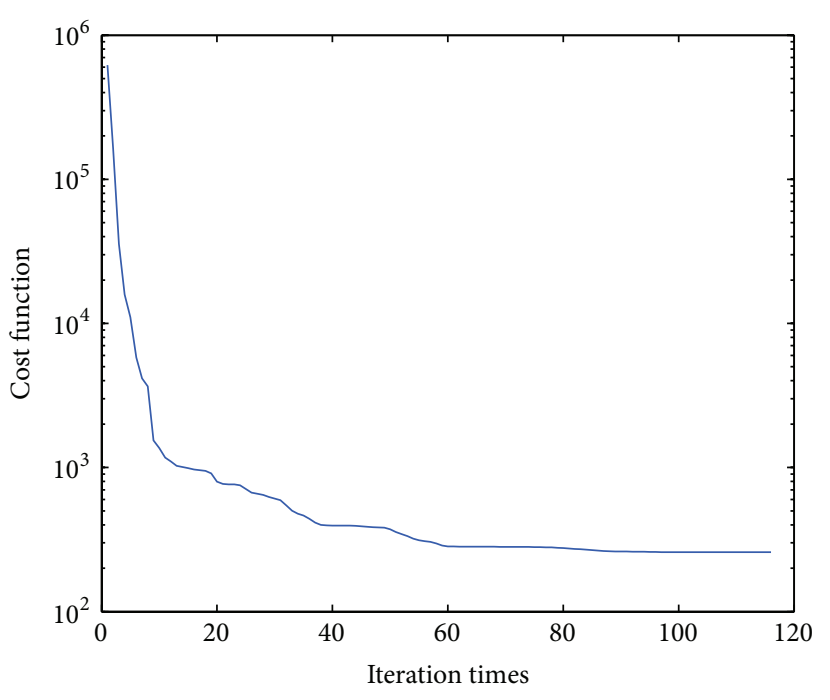

FiguRE 3: Surface temperature error of billet.

TABLE 1: Determination of an optimal value stopping parameter.

\begin{tabular}{lcc}
\hline eps & Iteration number & Cost function value \\
\hline 1 & 42 & 394.80 \\
0.1 & 115 & 258.15 \\
0.01 & 170 & 258.15 \\
\hline
\end{tabular}

TABLE 2: Physical parameters of steel grade.

\begin{tabular}{lc}
\hline Items & Parameters \\
\hline Specific heat & $540(\mathrm{~J} /(\mathrm{kg} \cdot \mathrm{k}))$ \\
Density & $7250\left(\mathrm{~kg} / \mathrm{m}^{3}\right)$ \\
Thermal conductivity & $30(\mathrm{~W} / \mathrm{m} \cdot \mathrm{K})$ \\
\hline
\end{tabular}

\section{Simulation Experiment}

To test the effectiveness of the method in this paper, the simulation experiments are done in actual plant data. The experiment result proves the effectiveness of the method in this paper. Physical parameters of steel grade are given in Table 2.

Example 1. Experimental parameters are shown as

$$
\begin{aligned}
& y_{\text {aim }}= \begin{cases}1173.16(\mathrm{~K}), & t<10(\mathrm{~s}), \\
1273.16(\mathrm{~K}), & t \geq 10(\mathrm{~s}),\end{cases} \\
& k=\frac{7250 \times 540}{50}, \quad \text { eps }=1 \times 10^{-1}, \\
& u(x, 0)=1173.16 \mathrm{~K}, \quad L=0.25 \mathrm{~m}, \\
& T=20 \mathrm{~s}, \quad u^{*}=0, \quad \alpha=1 \times 10^{-7} .
\end{aligned}
$$

In this example, the objective temperature changes from $1173.16(\mathrm{~K})$ to $1273.16(\mathrm{~K})$, when time is equal to 10 seconds. Figure 4 shows that the actual temperature can follow the objective temperature very well, so the quality of billet can be ensured. From Figure 5, we can know that the maximum 


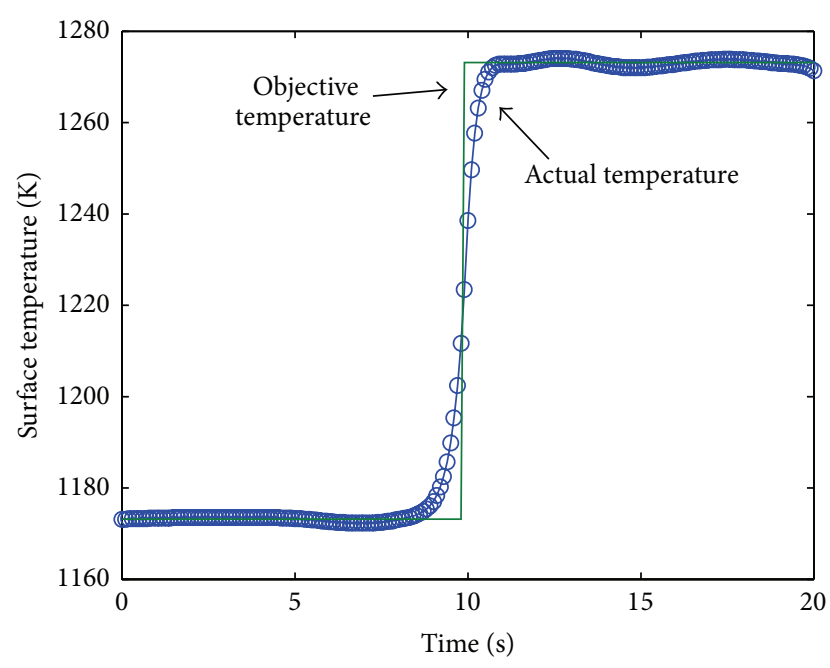

FIgURE 4: Surface temperature of billet.

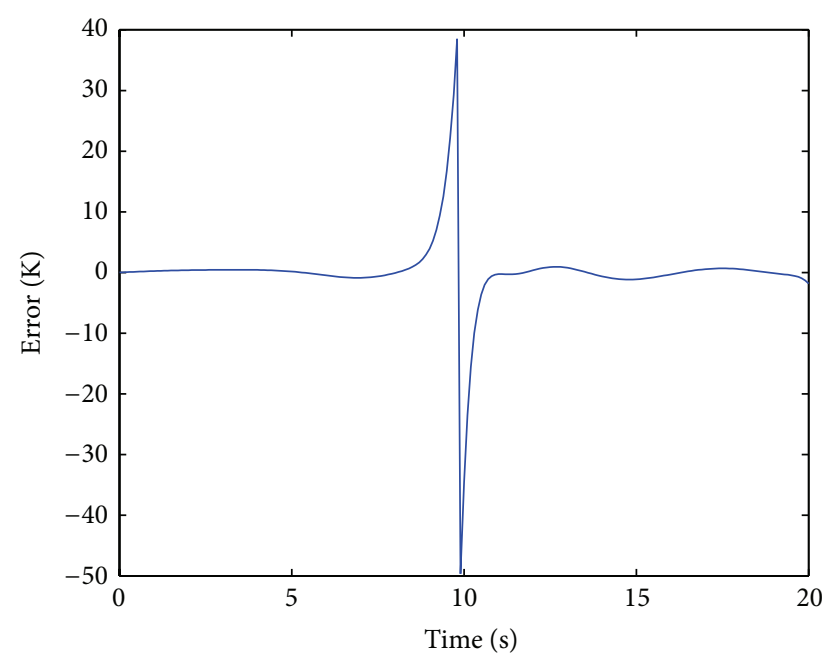

FIGURE 5: Surface temperature error of billet.

error is about $50(\mathrm{~K})$. The temperature error is very small in most of the time. Based on technological requirements in continuous casting process, the behaviour of the surface temperature ensures that the billet has a good quality. Figure 6 shows the controlled variable. Because of penalty of controlled variable, the controlled variable cannot be very large. When the controlled variable is less than zero, this process is a heating process. When the controlled variable is larger than zero, this process is a cooling process.

Example 2. Experimental parameters are shown as

$$
\begin{aligned}
& y_{\text {aim }}=1173.16+100 \sin \left(\frac{\pi}{10} t\right)(\mathrm{K}) \\
& k=\frac{7250 \times 540}{50}, \quad \text { eps }=1 \times 10^{-1}, \\
& u(x, 0)=1173.16 \mathrm{~K}, \quad L=0.25 \mathrm{~m}, \\
& T=20 \mathrm{~s}, \quad u^{*}=0, \quad \alpha=1 \times 10^{-7} .
\end{aligned}
$$

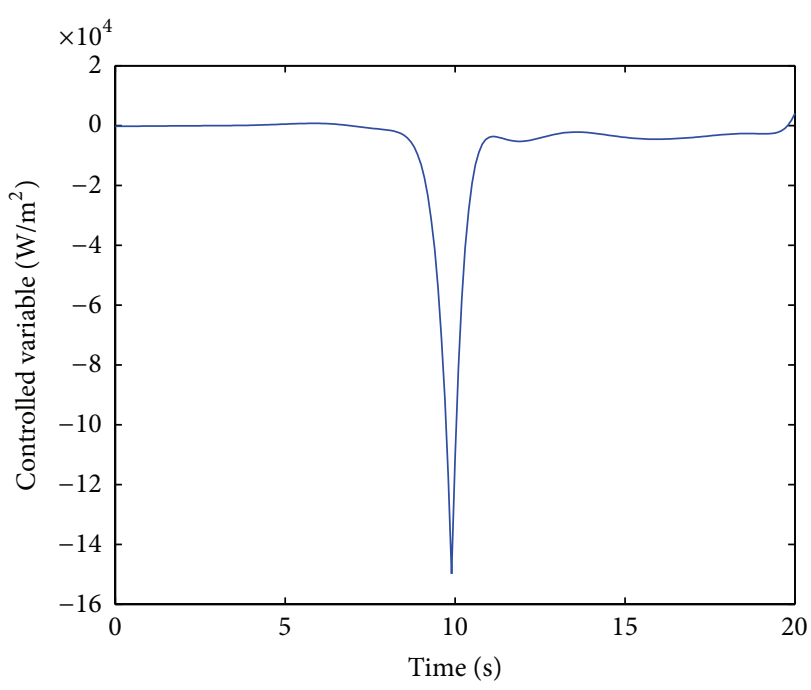

Figure 6: Controlled variable.

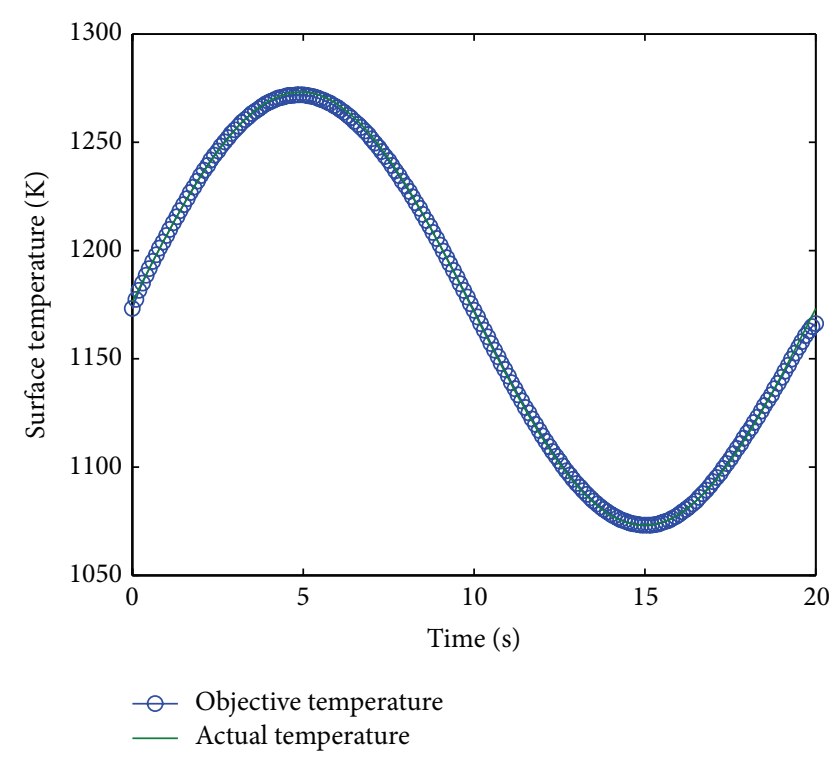

FIGURE 7: Surface temperature of billet.

In this example, the objective temperature is a sinusoidal curve which is continuous function. Figure 7 shows that the actual temperature can follow the objective temperature very well, so the quality of billet can be ensured. From Figure 8, we can know that the maximum error is about $8(\mathrm{~K})$. Because the objective temperature is a sinusoidal curve, the maximum error becomes smaller than Example 1. The temperature error is very small in most of the time. Based on technological requirements in continuous casting process, the behaviour of the surface temperature ensures that the billet has a good quality. Figure 9 shows the controlled variable. Because of a penalty of controlled variable, the controlled variable cannot be very large.

Example 3. Experimental parameters are shown as

$$
\begin{gathered}
y_{\text {aim }}=1673.16(\mathrm{~K}), \\
k=\frac{7250 \times 540}{50}, \quad \text { eps }=1 \times 10^{-1},
\end{gathered}
$$




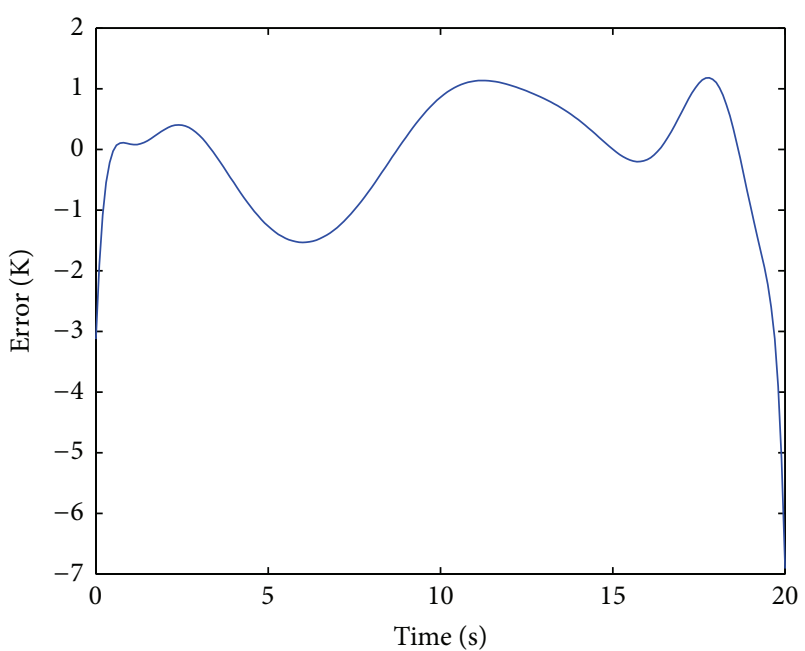

FIGURE 8: Surface temperature error of billet.

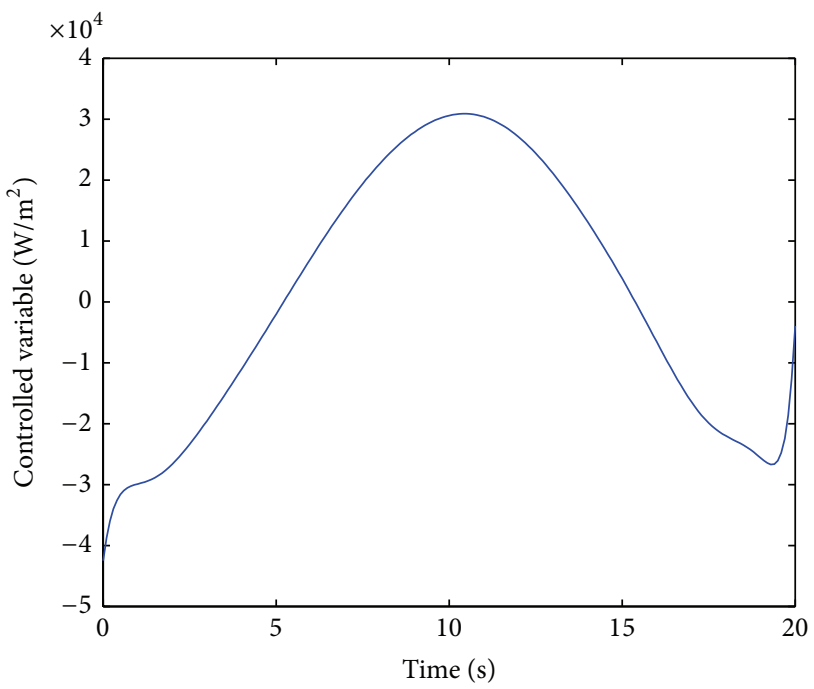

FIGURE 9: Controlled variable.

$u(x, 0)=1173.16 \mathrm{~K}, \quad L=0.25 \mathrm{~m}$,

$T=20 \mathrm{~s}, \quad u^{*}=0, \quad \alpha=1 \times 10^{-7}$.

In this example, the objective temperature is $1673.16(\mathrm{~K})$, but the initial temperature of billet is $1844.16(\mathrm{~K})$. Figure 10 shows that the actual temperature can follow the objective temperature very well. While the billet quality can be ensured, the surface temperature of billet can approach the objective temperature rapidly. From Figure 11, we can know that the maximum error is about $160(\mathrm{~K})$. Due to the penalties of control variable, the actual temperature cannot step to the objective temperature. Figure 12 shows the controlled variable. Because of penalty of controlled variable, the controlled variable cannot be very large.

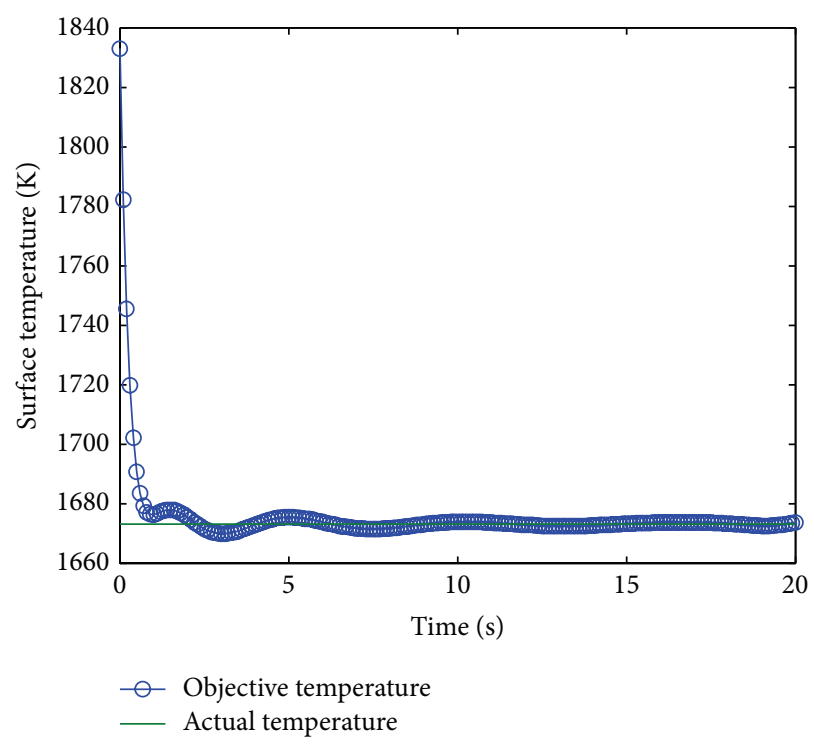

FIgURE 10: Surface temperature of billet.

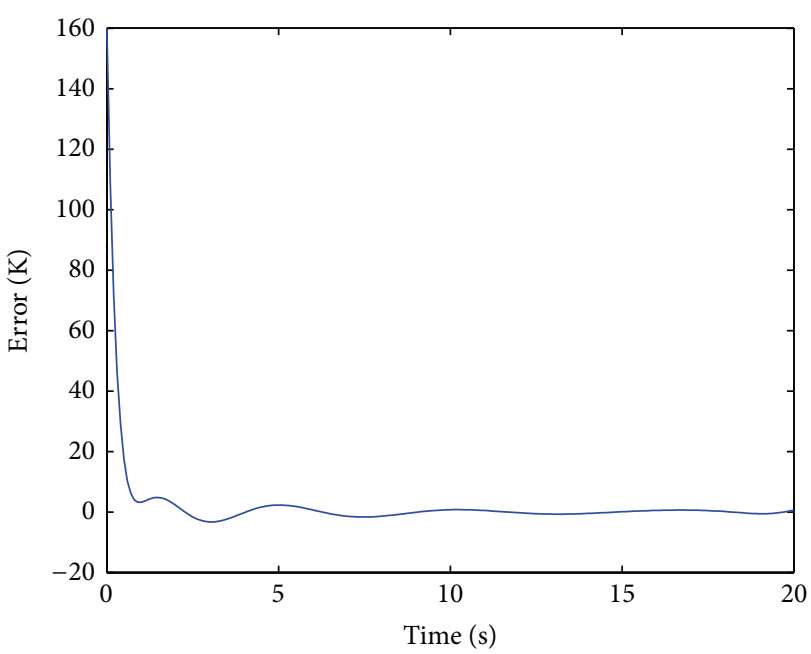

FIGURE 11: Surface temperature error of billet.

\section{Conclusions}

In order to solve optimization problem for set-point values in secondary cooling process, this paper applies first optimization and then discrete method to this optimal problem. We prove that the gradient of cost function is Lipschitz continuous. Base on this, we propose an improved conjugate algorithm to solve this problem and prove that this algorithm is convergent.

\section{Conflict of Interests}

The authors declare that there is no conflict of interests regarding the publication of this paper. 


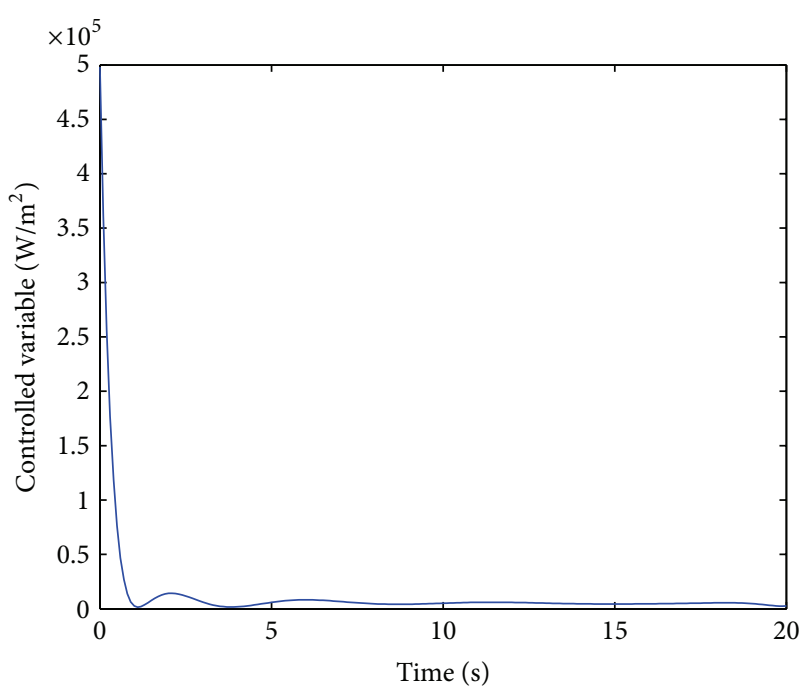

Figure 12: Controlled variable.

\section{Acknowledgments}

The authors wish to thank the reviewers for their careful reading and very helpful feedback, which helped improve the paper as well as the important guiding significance to their researches. This work was supported by the National Natural Science Foundation of China (61333006, 61473074) and the Fundamental Research for Central Universities (N120708001).

\section{References}

[1] V. Bratu, C. Mortici, C. Oros, and N. Ghiban, "Mathematical model of solidification process in steel continuous casting taking into account the convective heat transfer at liquid-solid interface," Computational Materials Science, vol. 94, pp. 2-7, 2014.

[2] A. V. Lotov, G. K. Kamenev, V. E. Berezkin, and K. Miettinen, "Optimal control of cooling process in continuous casting of steel using a visualization-based multi-criteria approach," Applied Mathematical Modelling, vol. 29, no. 7, pp. 653-672, 2005.

[3] M. Bergounioux and K. Kunisch, "Primal-dual strategy for state-constrained optimal control problems," Computational Optimization and Applications, vol. 22, no. 2, pp. 193-224, 2002.

[4] W. Liu and N. Yan, Adaptive Finite Element Methods for Optimal Control Governed by PDEs, Science Press, Beijing, China, 2008.

[5] M. Hinze, R. Pinnau, M. Ulbrich, and S. Ulbrich, Optimization with PDE Constraints, Springer, 2009.

[6] J. Stafford, E. Walsh, and V. Egan, "A study on the flow field and local heat transfer performance due to geometric scaling of centrifugal fans," International Journal of Heat and Fluid Flow, vol. 32, no. 6, pp. 1160-1172, 2011.

[7] M. Renardy, W. J. Hursa, and J. A. Nohel, Mathematical in Viscoelasticity, Wiley, 1987.

[8] D. Hömberg, K. Krumbiegel, and J. Rehberg, "Optimal control of a parabolic equation with dynamic boundary condition," Applied Mathematics and Optimization, vol. 67, no. 1, pp. 3-31, 2013.
[9] M. M. Kostreva and A. L. Ward, "Optimal control of a system governed by an elliptic partial differential equation," Journal of Computational and Applied Mathematics, vol. 114, no. 1, pp. 173187, 2000.

[10] H. Y. Li, "Estimation of thermal properties in combined conduction and radiation," International Journal of Heat and Mass Transfer, vol. 42, no. 3, pp. 565-572, 1998.

[11] K. H. Lee, S. W. Baek, and K. W. Kim, "Inverse radiation analysis using repulsive particle swarm optimization algorithm," International Journal of Heat and Mass Transfer, vol. 51, no. 11-12, pp. 2772-2783, 2008.

[12] M. H. Farag, F. H. Riad, and W. A. Hashem, "A penalty/MPQI method for constrained parabolic optimal control problems," International Journal of Computer Science, vol. 2, pp. 635-648, 2014.

[13] M. Kaya and A. Erdem, "Simultaneous reconstruction of the source term and the surface heat transfer coefficient," Mathematical Methods in the Applied Sciences, vol. 11, pp. 176-186, 2012.

[14] A. Hasanov, "Simultaneous determination of source terms in a linear parabolic problem from the final overdetermination: weak solution approach," Journal of Mathematical Analysis and Applications, vol. 330, no. 2, pp. 776-779, 2007.

[15] A. Hasanov, "An inverse source problem with single Dirichlet type measured output data for a linear parabolic equation," Applied Mathematics Letters, vol. 24, no. 7, pp. 1269-1273, 2011.

[16] A. Hasanov and B. Pektaş, "Identification of an unknown time-dependent heat source term from overspecified Dirichlet boundary data by conjugate gradient method," Computers \& Mathematics with Applications, vol. 65, no. 1, pp. 42-57, 2013.

[17] C. A. Santos, J. A. Spim, and A. Garcia, "Mathematical modeling and optimization strategies (genetic algorithm and knowledge base) applied to the continuous casting of steel," Engineering Applications of Artificial Intelligence, vol. 16, no. 5-6, pp. 511-527, 2003.

[18] B. You, M. Kim, D. Lee, J. Lee, and J. S. Lee, "Iterative learning control of molten steel level in a continuous casting process," Control Engineering Practice, vol. 19, no. 3, pp. 234-242, 2011.

[19] N. Cheung, C. A. Santos, J. A. Spim, and A. Garcia, "Application of a heuristic search technique for the improvement of spray zones cooling conditions in continuously cast steel billets," Applied Mathematical Modelling, vol. 30, no. 1, pp. 104-115, 2006.

[20] I. E. Livieris and P. Pintelas, "Globally convergent modified Perry's conjugate gradient method," Applied Mathematics and Computation, vol. 218, no. 18, pp. 9197-9207, 2012. 


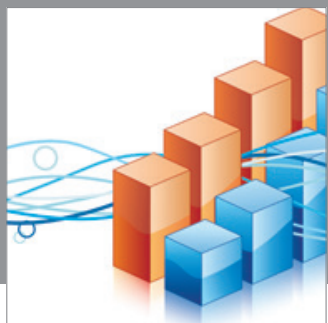

Advances in

Operations Research

mansans

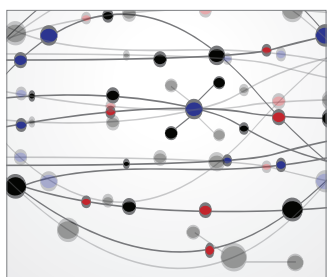

The Scientific World Journal
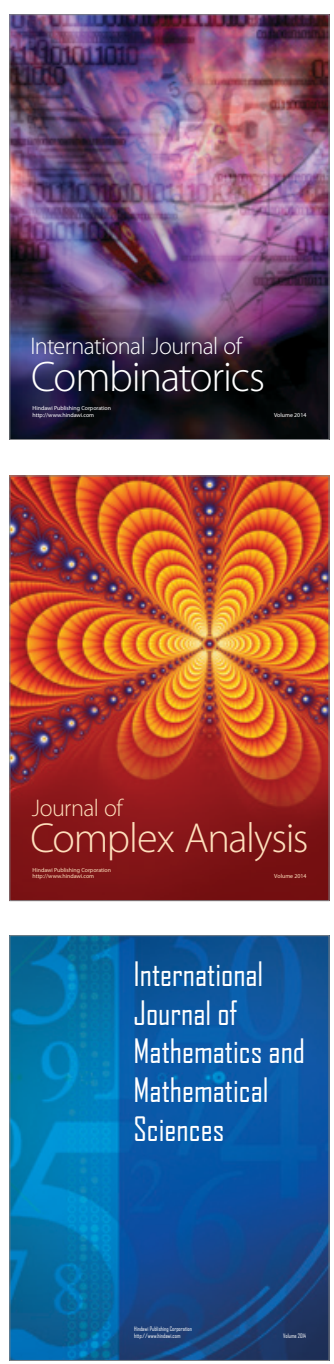
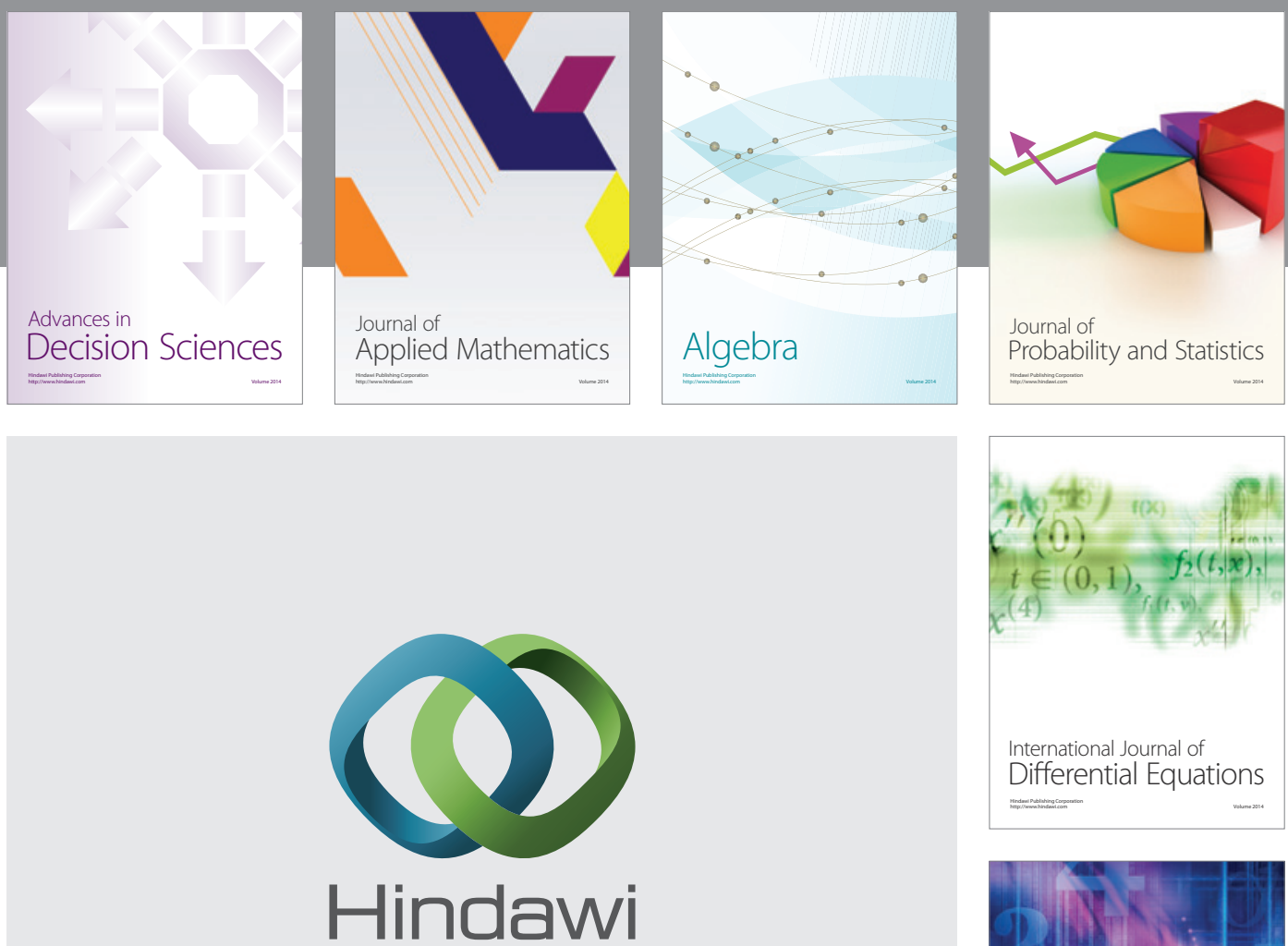

Submit your manuscripts at http://www.hindawi.com
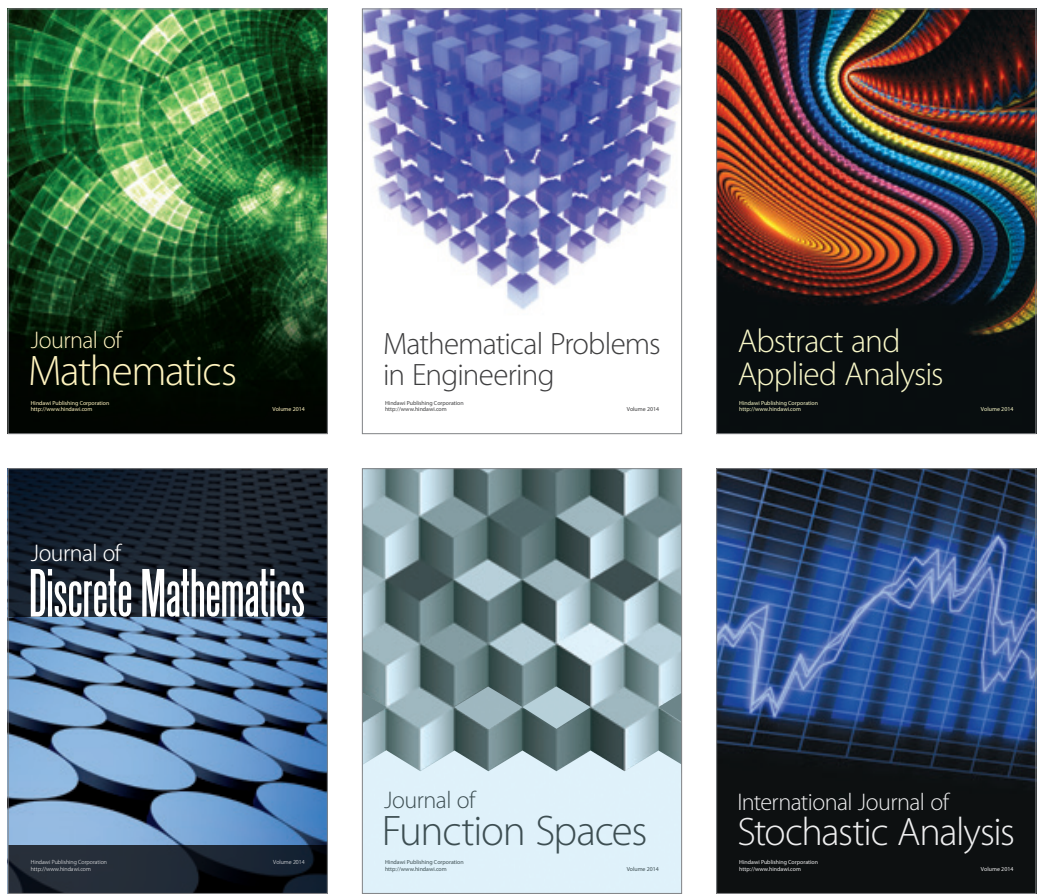

Journal of

Function Spaces

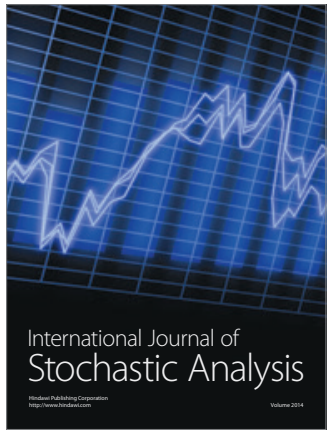

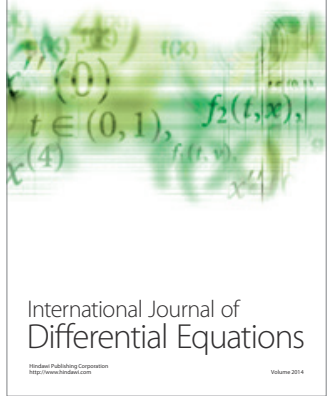
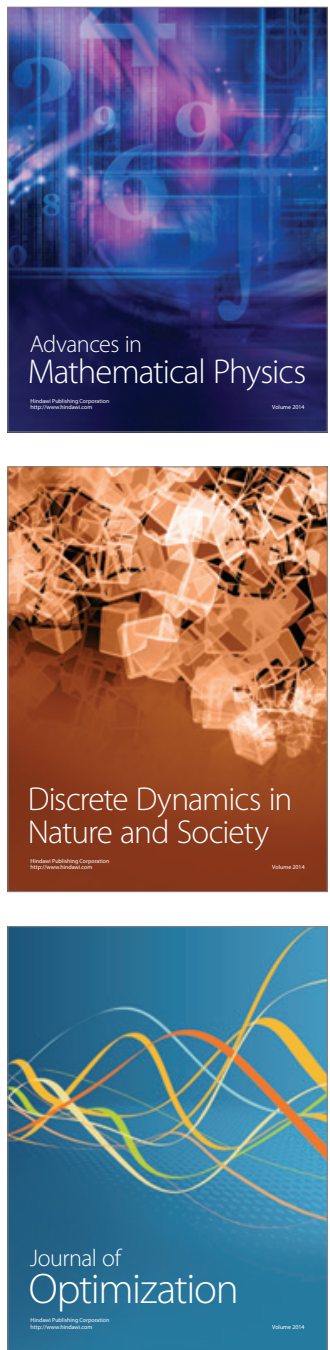